\title{
ANÁLISIS DE LA RESOLUCIÓN DE PROBLEMAS DE FÍSICA EN SECUNDARIA Y PRIMER CURSO UNIVERSITARIO EN CHILE
}

\author{
Becerra labra, Carlos ${ }^{1}$; Gras-Martí, Albert ${ }^{2}$ y Martínez-Torregrosa, Joaquín ${ }^{3}$ \\ ${ }^{1}$ Instituto de Física y Matemática. Universidad de Talca. Chile \\ ${ }^{2}$ Departament de Física Aplicada. Facultat de Ciències. Universitat d'Alacant \\ ${ }^{3}$ Departament de Didàctica General i Didàctiques Específiques. Universitat d'Alacant
}

\begin{abstract}
Resumen. La aspiración actual de la educación científica de enseñar a las personas cómo enfrentarse a problemas, de desarrollar destrezas de alto nivel intelectual al mismo tiempo que conocimientos específicos queda reducida en la enseñanza habitual de la física a la resolución de problemas de «lápiz y papel» al final de los temas. Sin embargo, el fracaso generalizado de los alumnos en esta actividad requiere poner en cuestión si, de verdad, se les está enseñando a resolver problemas. El análisis realizado de textos y profesores pone en evidencia que no se les enseña a resolver problemas, sino que se les explican soluciones ya hechas, transmitiendo serias deficiencias actitudinales y metodológicas que hacen enormemente difícil que puedan tener éxito ante nuevos problemas.
\end{abstract}

Palabras clave. Resolución de problemas de física, enseñanza de la física.

Summary. One of the expectations in Science Education nowadays is to teach people how to solve problems, to develop high-level intellectual skills at the same time that specific knowledge is acquired. In common practice of Physics Education, this objective is usually limited to solving «paper and pencil» problems appearing at the end of each book chapter. However, the widespread failure of students in this activity forces us to question if, indeed, we are teaching them how to solve problems. An analysis of both textbooks and teachers' practices shows that students are not taught how to solve problems but, instead, ready-made solutions are just explained to the students. This procedure transmits serious deficiencies both in attitudinal and methodological terms, which makes it very difficult for them to tackle new problems.

Keywords. Physics problem solving, physics education.

\section{LA ENSENANZA DE LA RESOLUCIÓN DE PROBLEMAS DE «LÁPIZ Y PAPEL» EN CUES- TIÓN: ¿DE VERDAD SE ENSEÑA A RESOLVER PROBLEMAS?}

La enseñanza de las ciencias en el nivel universitario y preuniversitario está actualmente en un proceso de desarrollo y cambio en todo el mundo (Tiberghien et al., 1998; Gil Pérez y Vilches, 1999). Éste se debe, en parte, a las evidencias aportadas por la investigación sobre el fracaso generalizado de buena parte del alumnado en el aprendizaje de las ciencias y también a las demandas planteadas por la sociedad a una educación que debería favorecer una alfabetización científica del ciudadano de este nuevo milenio (AAAS, 1989), y una preparación para la nuevas necesidades de los puestos de trabajo en un contexto caracterizado por una disminución de las tareas rutinarias (paulatinamente automatizadas) y un aumento de las destrezas de alto nivel intelectual. Todos los estudiantes, y no sólo unos pocos, necesitan aprender cómo pensar, razonar y comunicar eficazmente, cómo solucionar problemas complejos, y trabajar con grandes cantidades de datos, seleccionando los pertinentes para la toma de decisiones. No sólo es importante la comprensión profunda del contenido conceptual de las distintas materias, sino también (y simultáneamente) el desarrollo de destrezas complejas de pensamiento necesarias para desenvolverse competentemente en dichas materias (Black, 2001; NRC, 2001, p. 22).

Todas estas tendencias pueden resumirse en la aspiración de enseñar a los estudiantes a enfrentarse a situaciones problemáticas, a resolver problemas. Como explícitamente señalan los National Science Education Standards (1996): «[...] en todos los niveles, la educación científica debe basarse en la metodología de la investigación». 
$\mathrm{Y}$, aunque tomarse seriamente este objetivo implica organizar toda la enseñanza de las ciencias, y de la física en particular, en torno al tratamiento de problemas fundamentales, donde la resolución de problemas de «papel y lápiz» adquiere el estatus de «situaciones de puesta a prueba de los conceptos y modelos inventados para avanzar en la solución a problemas más amplios» (Martínez Torregrosa et al., 1994; Gil et al., 1999; Martínez Torregrosa et al., 2003; Verdú et al., 2002), en la realidad de la enseñanza habitual, el intento de enseñar a los alumnos a resolver problemas suele limitarse a la resolución de enunciados típicos al final de los temas. Tiene mucho interés, por tanto, analizar (al igual que se hizo anteriormente en otros países (Gil et al., 1987)) en qué medida se está utilizando esta actividad característica de las clases de física en un sentido coherente con las aspiraciones de la educación científica actual.

Como profesores de materias científicas sabemos que la resolución de problemas de «lápiz y papel» es una actividad a la que se suele dedicar mucho tiempo, tanto dentro como fuera del aula. Ello responde, claro está, a que se la considera una actividad privilegiada de aprendizaje (para «aclarar, aplicar o movilizar» los conceptos) y de evaluación, como indica su preponderancia en los exámenes habituales. Sin embargo, basta reflexionar mínimamente sobre nuestra experiencia como profesores de secundaria y de los primeros niveles universitarios para constatar el fracaso generalizado de los alumnos cuando se enfrentan por sí solos a un problema que se separe, aún ligeramente, de los resueltos en clase. ¿Cómo es posible que, a pesar del tiempo dedicado a las colecciones de problemas, se produzca esa aparente incapacidad para enfrentarse a nuevos problemas?

Desde nuestro punto de vista, superar esta situación requiere abandonar posibles justificaciones autoexculpatorias (los estudiantes no estudian, no saben aplicar los conceptos, no dominan las matemáticas...) y realizar un análisis en profundidad sobre lo que se hace habitualmente en las aulas para enseñar a resolver problemas que pongan en cuestión hasta lo más obvio: ¿De verdad estamos enseñando a nuestros alumnos a enfrentarse a problemas? ¿Estamos generando oportunidades adecuadas para que aprendan qué hacer, cómo pensar, ante un problema?

La respuesta racional a estas preguntas requiere, al menos, dos pasos. En primer lugar, clarificar qué entender por problema y qué debería caracterizar su proceso de resolución. Esto permitirá, en segundo lugar, plantearnos qué tipo de evidencias deberíamos conseguir para decidir en qué medida lo que se hace habitualmente en las aulas es coherente con lo que debería hacerse para enseñar a enfrentarse y resolver problemas.

\section{¿QUÉ ENTENDER POR PROBLEMA? ¿QUÉ DEBERÍA CARACTERIZAR SU PROCESO DE RESOLUCIÓN?}

Hace tiempo que los investigadores en la resolución de problemas de «lápiz y papel» (Krulik y Rudnik, 1980; Gil Pérez et al., 1988a) señalaron las limitaciones de las investigaciones que no se preguntan, en general, qué es un problema. Existe un acuerdo entre quienes sí han abordado la cuestión, en considerar un problema como una situación que presenta dificultades para las cuales no hay soluciones evidentes (Hudgins, 1966; Hayes, 1981; Gil Pérez y Martínez Torregrosa, 1983; Bodner y McMillen, 1986). La definición de Krulik y Rudnik (1980) resume bien este consenso: «Un problema es una situación, cuantitativa o no, de la que se pide una solución, para la cual los individuos implicados no conocen medios o caminos evidentes para obtenerla.» Del mismo modo, se habla de «umbral de problematicidad» para cada persona (Jansweijer et al., 1990), por encima del cual se puede decir que una situación constituye un verdadero problema para la persona en cuestión (y no un ejercicio, que no genera incertidumbre).

Precisamente, las ideas anteriores permiten criticar parcialmente la línea de investigación basada en el estudio comparativo de cómo resuelven problemas estándar cerrados, habituales en la enseñanza de la física, los expertos y los novatos. Dichas investigaciones buscan conocer como patrón empírico los modos procedimentales de ambos, identificar las diferencias y tratar de reforzar el comportamiento experto $-\mathrm{y}$ disminuir el novato- mediante la enseñanza-aprendizaje. Aunque el conocimiento de los procedimientos utilizados por los expertos y novatos, así como el del funcionamiento de las memorias de largo y corto plazo, y de la memoria sensorial, tiene un valor intrínseco evidente, presentan limitaciones claras. En efecto, al partir de problemas estándar cerrados, los expertos están trabajando por debajo de su umbral de problematicidad, con una seguridad sólo posible en quien conoce el camino de antemano. Podemos encontrarnos, pues, propugnando estrategias que consideran los problemas como no-problemas, como algo que se sabe hacer, lo que reforzaría la actitud habitual de los estudiantes de «reconocer o abandonar». Consideramos, pues, más productivo -y coherente con las aspiraciones de la educación científica actual- plantearnos qué hacen los científicos cuando se enfrentan a lo que para ellos constituye un verdadero problema, cuando se enfrentan a una situación problemática.

Dicho de otro modo, la actividad científica, la investigación, es un proceso en el que personas se enfrentan a situaciones problemáticas de interés, abiertas, y los aspectos que caracterizan esta forma de producir y aceptar conocimientos, de avanzar en los problemas, deberían estar presentes (en la medida de lo posible en el contexto escolar) también en el proceso de enseñanza-aprendizaje de la resolución de problemas en nuestras aulas.

No es posible realizar aquí un análisis mínimamente exhaustivo de la actividad científica. Simplemente nos referiremos a algunas características, que consideramos pertinentes para el tema que nos ocupa, sobre las que existe un consenso amplio entre los epistemólogos, historiadores (Chalmers, 1984; Harré, 1986) e investigadores en didáctica de las ciencias desde la perspectiva del «realismo moderado» (Longbottom y Butler, 1999; Osborne, 1996; Fernández et al., 2002): 
- Los problemas científicos son, en general, inicialmente «situaciones problemáticas confusas». Los problemas no vienen «dados», son situaciones que tienen interés (por distintas causas) pero que requieren ser simplificadas, modelizadas, definidas (operativizándolas, precisando cuál es el problema, qué se busca), partiendo, por supuesto, de los conocimientos que se poseen en el campo específico de la investigación. Es necesario «dar forma» a las situaciones problemáticas de interés, tomando decisiones para transformarlas en «investigables».

- El avance en el problema está guiado por hipótesis, por «tentativas de respuesta», que deben ser puestas a prueba lo más rigurosamente posible. El contexto hipotético-deductivo es característico de la actividad científica creativa, como señala Hempel (1976): «no se llega al conocimiento científico aplicando un proceso inductivo de inferencia a partir de datos recogidos con anterioridad, sino fundamentalmente intentando construir respuestas posibles a los problemas estudiados y sometiendo después las hipótesis a contrastación».

- Las investigaciones no se encuentran, en general, con los «datos» como punto de partida, sino, repetimos, con situaciones problemáticas confusas: las magnitudes que se consideran relevantes para avanzar en el problema, los datos, se eligen en función de la acotación del problema y de las hipótesis formuladas.

- Puesto que no se razona en términos de certeza, ni el conocimiento científico se descubre «mirando directamente a la naturaleza», sino en términos de hipótesis, es necesario dudar sistemáticamente de los resultados obtenidos y de todo el proceso de resolución. Ello obliga a interpretar y contrastar los resultados, mediante su consistencia con las hipótesis formuladas, a probar caminos distintos para ver si se obtiene lo mismo, a revisar la coherencia global con lo conocido en ese campo o a ver si lo hecho abre y permite avanzar en nuevos problemas de interés. Esto puede conducir a revisar las hipótesis, la estrategia de resolución o, incluso, a reformular el problema de forma distinta.

Enseñar, pues, a los estudiantes a resolver problemas implica -desde nuestra línea de investigación-desarrollar la enseñanza y el aprendizaje en un contexto problematizado, donde existan sistemática y reiteradamente oportunidades de poner en práctica las formas de pensamiento y acción de la actividad científica. Desde 1983, disponemos de un modelo para la enseñanza de resolución de problemas basado en esta concepción (Gil y Martínez Torregrosa, 1983) y de resultados obtenidos con su puesta en práctica en las aulas (Gil et al., 1988b; Ramírez et al., 1994; Furió y Reyes, 1990). Estamos ahora en disposición de analizar en qué medida se enseña a resolver problemas de física a los estudiantes chilenos de último curso de secundaria (17-18 años) y primer curso universitario (18-19 años).

\section{HIPÓTESIS Y DISEÑOS EXPERIMENTALES}

Según lo anterior, una enseñanza de la resolución de problemas de física que preparara a los estudiantes a enfrentarse y resolver problemas, debería contemplar ${ }^{1}$ los siguientes aspectos (Cuadro I).

Indicadores de una resolución de problemas de física coherente con la metodología científica.

1. La presentación de enunciados no directivos (tipo ejercicios), es decir, que no contengan de un modo exhaustivo condiciones, datos o apartados que indiquen los pasos a seguir antes de haber planteado siquiera qué es lo que se busca. Enunciados que favorezcan, en vez de obstaculizar, la resolución como indagación científica, tanto en los problemas resueltos como en los problemas propuestos.

2. La realización de un planteamiento cualitativo de la situación, imaginando la situación física, intentando acotar y definir de manera precisa el problema, dejando explícito qué es lo que se trata de determinar y en qué condiciones se va a resolver.

3. La formulación de hipótesis sobre los factores de los que puede depender la magnitud buscada y sobre la forma de esta dependencia, incluyendo, en particular, casos límites de fácil interpretación física que necesariamente debería contemplar el resultado.

4. La elaboración, con carácter tentativo, de posibles estrategias de resolución antes de proceder a ésta, para posibilitar una contrastación rigurosa de la(s) hipótesis y mostrar la coherencia con el cuerpo de conocimientos de que se dispone. No presentan la estrategia como algo evidente o seguro.

5. La resolución del problema como la puesta en práctica de la estrategia planteada, verbalizando lo que se hace y evitando operativismos carentes de significación física.

6. El análisis de los resultados obtenidos a la luz de la(s) hipótesis elaborada(s) y, en particular, de los casos límite considerados. Siempre poniendo en duda los resultados obtenidos.

7. La consideración de las perspectivas abiertas tras la resolución, contemplando, por ejemplo, la posibilidad de abordar el problema a un nivel de mayor complejidad o de abordar nuevas situaciones de interés práctico o teórico. Esta reflexión sobre nuevas perspectivas debería incluir una breve recapitulación sobre las dificultades encontradas y la forma en que se han superado (lo que contribuye a mejorar la capacidad para enfrentarse a nuevos problemas). 
Es necesario resaltar que algunas de estas características, como el planteamiento cualitativo inicial, la elaboración de posibles estrategias de resolución o el análisis de los resultados, son consideradas esenciales desde otras concepciones sobre resolución de problemas (Reif, 1983; Gil Pérez et al., 1988a; Maloney, 1994; Perales y Cañal, 2000), y su carencia no sólo evidenciaría que no se abordan los problemas como tales, sino que marcarían deficiencias «absolutas» desde cualquiera de las tendencias actuales de la investigación didáctica sobre resolución de problemas.

Nuestra hipótesis es que el fracaso constatado no puede ser fruto de la casualidad o de la incapacidad de los estudiantes, sino que debe estar relacionado con lo que se hace en clase. Dicho de otro modo: en la enseñanza habitual no se enseña a resolver problemas como tales, como situaciones abiertas que requieren poner en práctica formas de pensamiento características del trabajo científico, sino como ejercicios de aplicación de la teoría que los estudiantes deben asimilar y reproducir.

Para contrastar nuestra hipótesis hemos procedido a comprobar en qué medida los indicadores de una resolución de los problemas de física coherente con la metodología científica están presentes en: $a$ ) los libros de texto de física usados habitualment; $b$ ) las prácticas docentes de los profesores; y $c$ ) en el modo de actuar de los estudiantes cuando resuelven problemas (que suelen reflejar el modelo que han vivido).

Hemos realizado un análisis de los problemas resueltos y los enunciados propuestos en los libros de texto más utilizados en las clases de física en la región chilena de Talca (26 de nivel universitario y 6 de secundaria, los más usados en los últimos diez años), con el objetivo de determinar en qué medida están presentes los indicadores de calidad citados en el cuadro I.

Para estudiar qué hacen y piensan los profesores de física, hemos utilizado cuatro instrumentos distintos, que permiten aumentar la fiabilidad de las conclusiones mediante la coherencia de la evidencia obtenida por caminos distintos. Estos instrumentos han sido:

1) Análisis de las críticas y objeciones planteadas por los profesores a un problema resuelto de un texto habitual (Resnick y Halliday, 1988) con serias deficiencias en los indicadores del cuadro I (Anexo I).

2) Análisis de la resolución didáctica ideal de los profesores, obtenida presentando, a los profesores (21 de secundaria y 10 universitarios), un enunciado habitual -claramente familiar para ellos- y pidiéndoles que lo resolvieran «didácticamente», de modo que quedaran reflejadas en el papel sus ideas sobre cómo habría que enseñarles a resolver problemas (Anexo II).

3) Análisis de los cuadernos de apuntes de alumnos de distintas clases y niveles ( 15 de secundaria y 18 universitarios). Como señala Gilbert (1980), los profesores somos un modelo que los estudiantes imitan, por ello hemos estudiado los apartados de los cuadernos en donde se recogen problemas resueltos de la misma forma que con los libros de texto.

4) Para afianzar e ilustrar los resultados cuantitativos, hemos realizado entrevistas individuales semiestructuradas, que han sido grabadas, a 21 profesores de secundaria y 10 de primer curso universitario, en 2 universidades. Las entrevistas siempre han empezado de la misma forma:

«Estamos realizando un estudio sobre cómo podríamos mejorar la resolución de problemas de los estudiantes, y nos interesan los conocimientos de profesores con experiencia. Es un hecho constatado que la gran mayoría de los alumnos fracasan ante un problema que se separe de los hechos en clase. ¿Qué podrías hacer en el aula para mejorar esta situación? ¿Qué recomendaciones darías a tus alumnos para mejorar su aprendizaje en resolución de problemas?» Tras la respuesta del entrevistado a estas preguntas abiertas, el entrevistador hacía nuevas preguntas dirigidas únicamente a clarificar y obtener más información sobre la concepción del proceso de resolución de problemas. Los protocolos así obtenidos han sido analizados según los indicadores expresados en el cuadro I para valorar en qué medida se encontraban presentes recomendaciones coherentes con los mismos o, por el contrario, con una concepción de los problemas como meros ejercicios de aplicación de la teoría, cuyo éxito sólo se ve afectado por falta de conocimientos teóricos y/o matemáticos, de entrenamiento (haber realizado muchos problemas) o inatención (lectura detenida del enunciado).

El último de los instrumentos que hemos utilizado para obtener evidencias sobre cómo se enseña a resolver problemas ha sido la resolución de dos problemas elementales y prototípicos (según el nivel académico «teórico» de la física en secundaria) de mecánica realizada por 40 alumnos de último curso de secundaria (los 10 que habían obtenido mejor calificación en Física en cuatro clases distintas) y 80 estudiantes, elegidos al azar, de primer curso de Ingeniería en Agronomía de la Universidad de Talca, después de haber cursado la parte de mecánica.

Los enunciados de los problemas fueron los siguientes:

Te pedimos que resuelvas los siguientes problemas, incluyendo los comentarios, datos, aclaraciones que consideres necesario.

1) Un estudiante está corriendo a la velocidad constante de $10 \mathrm{~m} / \mathrm{s}$; cuando está a $40 \mathrm{~m}$ de una moto, ésta parte del reposo con una aceleración de $0,9 \mathrm{~m} / \mathrm{s}^{2}$ en la misma dirección y sentido en que corre el estudiante. ¿Cuánto tiempo tarda el estudiante en alcanzar la moto? Determina el tiempo durante el cual el estudiante se mantiene por delante de la moto.

2) Un automóvil de $1.600 \mathrm{~kg}$ de masa se mueve a la velocidad de $35 \mathrm{~m} / \mathrm{s}$ en una calle plana. Si se aplica una fuerza de frenado constante de $5 \times 10^{3} \mathrm{~N}$ a las llantas del auto hasta detenerse, ¿qué distancia recorrerá el vehículo después de aplicar los frenos? 
Se trataba de mostrar cómo en la resolución de estudiantes con éxito se veían reflejadas las mismas deficiencias, respecto a los indicadores de calidad, que en libros y profesores, completando así la imagen de cómo se enseña a resolver problemas. Los estudiantes dispusieron de tiempo suficiente y en la valoración de las resoluciones de ambos problemas se eligió siempre la opción más desfavorable a nuestra hipótesis (si un indicador estaba presente en uno de los dos, se consideraba negativo para la hipótesis).

\section{PRESENTACIÓN Y ANÁLISIS DE RESULTADOS}

En la tabla I se muestran los resultados obtenidos al analizar textos, cuadernos de apuntes de alumnos y la resolución didáctica ideal de los profesores: $(\mathrm{S}=$ secundaria, $\mathrm{U}=$ universitaria). Como vemos, prácticamente la totalidad de los enunciados encontrados son cerrados, incluyendo condiciones, apartados, datos, etc., lo que parece indicar que textos y profesores no conciben los problemas como situaciones abiertas, inciertas, y su proceso de resolución como un proceso donde deben ponerse en práctica las formas de pensamiento y acción características de la forma en que se enfrentan los científicos a los problemas. Por el contrario, la inclusión casi única de este tipo de enunciados obstaculiza el desarrollo de dichas actividades intelectuales (como la formulación de hipótesis, la elaboración de estrategias con carácter tentativo, la duda de los resultados obtenidos...), y es una muestra de una concepción de los problemas como meros ejercicios de aplicación de la teoría.

Esta afirmación no se basa únicamente, claro está, en la omnipresencia de los enunciados cerrados, sino, como puede verse en la tabla I:

a) en la ausencia de un planteamiento o un análisis cualitativo de la situación antes de usar datos y fórmulas (sólo 1 de cada 4 profesores lo hacen en su resolución didáctica ideal, y está ausente en el 87,9\% de los cuadernos de los alumnos);

b) en la ausencia total de formulación de hipótesis sobre de qué y de qué forma dependerá aquello que se busca (de hecho, en la mayoría de los casos, 74,2 \% y 78,8 \% de los profesores y cuadernos, se desarrolló directamente la resolución sin haber procedido previamente a concretar cuál es el problema, qué se busca; en los libros, el tanto por ciento es menor $(50 \%)$ porque $(*)$ los enunciados son tan directivos que ya está totalmente precisado qué se busca);

c) en la ausencia de comentario alguno sobre el carácter tentativo de la estrategia $(0 \%)$. De hecho, en más de un $80 \%$ de los textos y resoluciones ideales de los profesores, no se plantea una estrategia antes de desarrollarla (una manera de proceder sólo posible cuando uno se enfrenta a un ejercicio, a una situación que no le genera incertidumbre, donde actúa con la seguridad de que lo que está haciendo es correcto de antemano);

d) coherentemente con esta concepción aproblemática de los problemas, no se duda del resultado obtenido
(100\% de las tres muestras), como indica la ausencia de cualquier tipo de prueba o comentario en este sentido sobre el resultado (a pesar de que un tercio de los profesores realizó una resolución literal, lo que favorece enormemente someter a pruebas el resultado). Tan sólo un pequeño porcentaje (en torno al $20 \%$ ) acompaña el resultado obtenido de algún comentario sobre la cuantía o significado físico del mismo, lo que hemos considerado positivamente como «interpretación del resultado» (pero sin dudar de que sea correcto);

$e$ ) en la inexistencia de intento alguno de aprovechar el resultado obtenido en un problema para plantear o resolver nuevos problemas relacionados. No sólo se ignora una característica del buen trabajo científico, sino que este modo de proceder es coherente con la idea de que lo importante «es hacer muchos problemas» (Tabla III), como si los enunciados habitualmente propuestos al final de los temas fueran esencialmente distintos entre sí. Sin embargo, basta pensar en los problemas de mecánica y plantearnos cuántos problemas esencialmente distintos se encuentran al final de los temas, ¿no sería mejor el tratamiento adecuado y en profundidad de unos pocos problemas seleccionados que las colecciones de problemas resueltos como no-problemas? La utilización de las grandes ideas fundamentales y el aprovechamiento de los resultados obtenidos en unos pocos problemas bien seleccionados para plantear otros que sólo suponen pequeños cambios reduciría el tiempo dedicado a tareas realizadas sin comprensión y por ensayo y error. Como señalan Leonard y otros (2002), resolver muchos problemas favorece y refuerza aproximaciones basadas en las fórmulas y un aprendizaje superficial.

En el anexo I se muestra, a modo de ejemplo, una resolución típica, extraída de un libro de física usado habitualmente en el primer curso universitario (Resnick y Halliday, 1988), con serias deficiencias en los indicadores del cuadro I: $a$ ) enunciado totalmente directivo; b) ausencia de planteamiento cualitativo destinado a imaginar la situación, cuál es el problema, qué condiciones se van a suponer...; $c$ ) presenta el proceso de resolución como un proceso lineal, sin incertidumbre (no se formulan hipótesis o intento alguno de especulación fundada sobre las posibles relaciones existentes entre los datos y lo que se pide; no elabora en forma explícita una posible estrategia de resolución antes de proceder a ésta; no fundamenta ni verbaliza lo que hace); $d$ ) no se duda del resultado obtenido, sólo se interpreta el signo negativo de la aceleración (para este caso, hemos considerado que «se interpretan los resultados obtenidos»). Incita a una manipulación inmediata de fórmulas, datos e incógnitas, dirigiendo a la ecuación apropiada, según la tabla. Esta forma de presentar didácticamente la resolución de problemas como meros ejercicios de aplicación de algo ya sabido, que no genera incertidumbre, se ve reflejada también en los resultados obtenidos del análisis de las «críticas y objeciones a un problema resuelto» (se trataba del mismo problema del anexo I) y de las entrevistas individuales que se muestran en las tablas II y III. 
Tabla I

Resultado del análisis de textos, resoluciones ideales de profesores y cuadernos de apuntes de alumnos.

\begin{tabular}{|c|c|c|c|c|c|c|c|c|c|}
\hline \multirow{2}{*}{$\begin{array}{l}\text { Valoración respecto a los indicadores de } \\
\text { una resolución de problemas coherente } \\
\text { con la metodología científica }\end{array}$} & \multicolumn{3}{|c|}{ Libros de texto } & \multicolumn{3}{|c|}{$\begin{array}{c}\text { Resolución didáctica ideal } \\
\text { (profesores) }\end{array}$} & \multicolumn{3}{|c|}{$\begin{array}{l}\text { Cuadernos de apuntes } \\
\text { (alumnos) }\end{array}$} \\
\hline & $\begin{array}{c}\mathrm{S} \\
(\mathrm{N}=6) \\
\mathrm{n}\end{array}$ & $\begin{array}{c}\mathrm{U} \\
(\mathrm{N}=26) \\
\mathrm{n}\end{array}$ & $\begin{array}{c}\mathrm{S}+\mathrm{U} \\
\%\end{array}$ & $\begin{array}{c}\mathrm{S} \\
(\mathrm{N}=21) \\
\mathrm{n}\end{array}$ & $\begin{array}{c}\mathrm{U} \\
(\mathrm{N}=10) \\
\mathrm{n}\end{array}$ & $\begin{array}{c}\mathrm{S}+\mathrm{U} \\
\%\end{array}$ & $\begin{array}{c}\mathrm{S} \\
(\mathrm{N}=15) \\
\mathrm{N}\end{array}$ & $\begin{array}{c}\mathrm{U} \\
(\mathrm{N}=18) \\
\mathrm{n}\end{array}$ & $\begin{array}{c}\mathrm{S}+\mathrm{U} \\
\%\end{array}$ \\
\hline \multicolumn{10}{|l|}{ I. Sobre el tipo de enunciados } \\
\hline $\begin{array}{l}\text { 1. No hacen objeción sobre el tipo de enun- } \\
\text { ciado, por ejemplo, que es totalmente direc- } \\
\text { tivo o presentan enunciados tipo ejercicio }\end{array}$ & 6 & 25 & 96,9 & --- & ---- & & 15 & 18 & 100 \\
\hline \multicolumn{10}{|l|}{ II. Sobre el planteamiento cualitativo } \\
\hline $\begin{array}{l}\text { 2. No expresan cualitativamente aspec- } \\
\text { tos de la situación física antes de usar } \\
\text { fórmulas }\end{array}$ & 5 & 17 & 68,8 & 18 & 4 & 71,0 & 15 & 14 & 87,9 \\
\hline $\begin{array}{l}\text { 3. No dejan explícito qué es lo que se pide } \\
\text { y en qué condiciones se va a realizar la } \\
\text { resolución }\end{array}$ & 4 & 12 & $\begin{array}{c}50,0 \\
(*)\end{array}$ & 19 & 4 & 74,2 & 14 & 12 & 78,8 \\
\hline \multicolumn{10}{|l|}{ III. Sobre la emisión de hipótesis } \\
\hline $\begin{array}{l}\text { 4. No emiten hipótesis sobre los factores } \\
\text { de los que puede depender la magnitud } \\
\text { buscada y la forma de esta dependencia }\end{array}$ & 6 & 26 & 100 & 21 & 10 & 100 & 15 & 18 & 100 \\
\hline \multicolumn{10}{|l|}{ IV. Sobre la elaboración de estrategias } \\
\hline $\begin{array}{l}\text { 5. No expresan en forma explícita una } \\
\text { posible estrategia de resolución antes de } \\
\text { proceder a ésta }\end{array}$ & 6 & 22 & 87,5 & 20 & 5 & 80,6 & 15 & 18 & 100 \\
\hline $\begin{array}{l}\text { 6. No expresan en forma explícita el carác- } \\
\text { ter tentativo de la estrategia }\end{array}$ & 6 & 26 & 100 & 21 & 10 & 100 & 15 & 18 & 100 \\
\hline \multicolumn{10}{|l|}{ V. Sobre la resolución del problema } \\
\hline $\begin{array}{l}\text { 7. No hacen la resolución como la puesta } \\
\text { en práctica de la estrategia planteada }\end{array}$ & 6 & 22 & 87,5 & 20 & 5 & 80,6 & 15 & 18 & 100 \\
\hline $\begin{array}{l}\text { 8. No hacen la resolución fundamentando } \\
\text { y verbalizando lo que se va haciendo }\end{array}$ & 4 & 19 & 71,9 & 19 & 4 & 74,2 & 15 & 18 & 100 \\
\hline $\begin{array}{l}\text { 9. No hacen una resolución literal antes de } \\
\text { introducir valores numéricos }\end{array}$ & 4 & 18 & 68,8 & 15 & 3 & 58,1 & 10 & 12 & 66,7 \\
\hline \multicolumn{10}{|l|}{ VI. Sobre el análisis de los resultados } \\
\hline 10. No interpretan los resultados obtenidos & 5 & 20 & 78,1 & 20 & 7 & 87,1 & 15 & 12 & 81,8 \\
\hline $\begin{array}{l}\text { 11. No contrastan (no dudan de) los resul- } \\
\text { tados obtenidos }\end{array}$ & 6 & 26 & 100 & 21 & 10 & 100 & 15 & 18 & 100 \\
\hline \multicolumn{10}{|l|}{$\begin{array}{l}\text { VII. Sobre la consideración de perspecti- } \\
\text { vas abiertas }\end{array}$} \\
\hline $\begin{array}{l}\text { 12. Tras la resolución, no contemplan la } \\
\text { posibilidad de abordar nuevas situaciones } \\
\text { de interés }\end{array}$ & 6 & 20 & 81,3 & 21 & 9 & 96,8 & 15 & 18 & 100 \\
\hline
\end{tabular}


Tabla II

Críticas y objeciones planteadas por los profesores a un problema resuelto habitual.

\begin{tabular}{|c|c|c|c|}
\hline & $\begin{array}{l}\mathbf{S}(\mathbf{N}=\mathbf{2 1}) \\
\mathbf{N}\end{array}$ & $\begin{array}{l}\mathbf{U}(\mathbf{N}=\mathbf{1 0}) \\
\mathbf{n}\end{array}$ & $\begin{array}{l}\mathbf{S}+\mathbf{U}(\mathbf{N}=\mathbf{3 1}) \\
\%\end{array}$ \\
\hline \multicolumn{4}{|l|}{$\begin{array}{l}\text { Respuestas que expresan objeciones o comentarios relacionados con los indicadores de } \\
\text { una resolución como una indagación científica: }\end{array}$} \\
\hline El enunciado es totalmente directivo (tipo ejercicio) & 0 & 2 & 6,5 \\
\hline La ausencia de un análisis cualitativo de la situación & 2 & 4 & 19,4 \\
\hline $\begin{array}{l}\text { La ausencia de formulación de hipótesis sobre los factores de los que puede depender la } \\
\text { magnitud buscada }\end{array}$ & 0 & 0 & 0 \\
\hline La ausencia de una estrategia de resolución antes de proceder a ésta & 2 & 3 & 16,1 \\
\hline No se expresa el carácter tentativo de la estrategia & 0 & 0 & 0 \\
\hline No se fundamenta lo que se hace & 1 & 1 & 6,5 \\
\hline La ausencia de un análisis de los resultados & 1 & 1 & 6,5 \\
\hline No se ponen en duda los resultados obtenidos & 0 & 0 & 0 \\
\hline $\begin{array}{l}\text { No se contempla el interés de abordar el problema a un nivel de mayor complejidad o } \\
\text { problemas relacionados }\end{array}$ & 0 & 0 & 0 \\
\hline \multicolumn{4}{|l|}{$\begin{array}{l}\text { Respuestas que plantean objeciones «de forma» o relacionadas con la concepción del } \\
\text { problema como ejercicio de aplicación, tales como: }\end{array}$} \\
\hline El enunciado es poco claro & 19 & 6 & 80,6 \\
\hline Falta de una resolución más explicada & 21 & 9 & 96,8 \\
\hline Falta de esquemas, dibujos, gráficos, etc. & 20 & 8 & 90,3 \\
\hline Aspectos relacionados con datos, unidades, etc. & 21 & 8 & 93,5 \\
\hline
\end{tabular}

A modo de ejemplo del tipo de críticas más comunes (las que se encuentran al final de la tabla II) al proceso de resolución del problema presentado (Anexo I): «Debería extraer y colocar de inmediato todos los datos dados en orden y de inmediato las incógnitas (lo que piden)». «Falta de un diagrama o dibujo de la situación y del sistema de coordenadas usado». «Explicar en forma más detallada la resolución», etc. Existe una ausencia casi total de críticas a las deficiencias y carencias de tipo metodológico y actitudinal que transmite a los alumnos, de un modo absolutamente claro, el problema presentado. Tanto si se concibe la resolución de problemas como algo semejante a una investigación, o si, meramente, se tiene presente la naturaleza tentativa, no lineal, de su proceso de resolución (¡algo que necesariamente han tenido que vivir los profesores cuando se han enfrentado a problemas que no sabían hacer!), es indiscutible la deformación metodológica de este tipo de «problemas resueltos». Sin embargo, los profesores parecen no estar preocupados o no ser conscientes de esta cuestión.

El análisis de las entrevistas, como se ve en la tabla III, confirma la afirmación anterior: sólo están presentes de manera significativa recomendaciones que asignan la culpa al modo de proceder de los estudiantes (no leen detenidamente el enunciado, no conocen la teoría, tienen deficiencias matemáticas) y manifiestan una creencia en que el avance sería automático y seguro si se dispusiera de dichos conocimientos. Los profesores suprimen o desconsideran el proceso de incertidumbre que es característico de la resolución de problemas. A modo de ejemplo, presentaremos parte de una entrevista de un profesor de física. Algunas de las recomendaciones (textuales) que daría a sus alumnos son: «En primer lugar tienen que estudiar bien la materia. Realicen muchos problemas, para ejercitarse. Lean bien el problema para entenderlo. Si no viene un diagrama o dibujo de la situación, hacerlo. Extraigan todos los datos (deben dejarlos todos en un mismo sistema de unidades) y las incógnitas del problema. Luego vean cuál(es) fórmula(s) sirve(n) para resolver el problema. Tengan mucho cuidado al despejar la incógnita de la(s) fórmula(s).»

Con la evidencia mostrada hasta aquí, podemos afirmar que, muy probablemente, los profesores presentan lo que son ejercicios para ellos, pero problemas para sus alumnos, como ejercicios de aplicación de la teoría, que sólo requieren un proceso lineal, sin incertidumbres. Nos atrevemos a sugerir que, aunque los mismos profesores hayan pasado varias horas intentando resolver un problema, una vez resuelto, lo presentan en clase escamoteando todo el proceso de incertidumbre, de dar vueltas, que es inherente al proceso de solución. Ante los ojos de los alumnos, la resolución de problemas aparece, pues, como un proceso lineal, fruto de una intuición inmediata, que, aparentemente, sólo está al alcance del profesor y algunos compañeros muy dotados. Además se les transmite que esa manera de proceder la alcanzará si realiza muchos problemas, sin enseñarle, en momento alguno, cómo enfrentarse actitudinal, metodológica y conceptualmente a dichos problemas. 
Tabla III

Análisis de los protocolos de las entrevistas a los profesores.

\begin{tabular}{|c|c|c|c|}
\hline & $\begin{array}{l}\mathbf{S}(\mathbf{N}=21) \\
\quad \mathbf{N}\end{array}$ & $\begin{array}{l}\mathbf{U}(\mathbf{N}=10) \\
\quad \mathbf{n}\end{array}$ & $\begin{array}{c}\mathbf{S}+\mathbf{U} \\
(\mathbf{N}=\mathbf{3 1}) \%\end{array}$ \\
\hline \multicolumn{4}{|l|}{$\begin{array}{l}\text { Respuestas que expresan formas de actuar y recomendaciones directamente relacionadas con } \\
\text { una concepción de los problemas como situaciones inciertas, tales como: }\end{array}$} \\
\hline $\begin{array}{l}\text { Hacer alguna referencia a que es normal que un problema genere incertidumbre, y que en el aula se } \\
\text { debe actuar en sintonía con esto }\end{array}$ & 0 & 0 & 0 \\
\hline $\begin{array}{l}\text { Plantear alguna objeción a los enunciados habituales, por ejemplo, que son totalmente directivos e } \\
\text { inducen al operativismo }\end{array}$ & 0 & 2 & 6,5 \\
\hline $\begin{array}{l}\text { Mencionar alguna recomendación sobre la necesidad de realizar un análisis cualitativo de la } \\
\text { situación antes de proceder a la resolución }\end{array}$ & 2 & 4 & 19,4 \\
\hline $\begin{array}{l}\text { Hacer alguna mención a la naturaleza tentativa del proceso de resolución (por ejemplo, que la } \\
\text { estrategia debe ser tomada como una hipótesis, el resultado debe ser puesto a prueba, etc.) }\end{array}$ & 0 & 0 & 0 \\
\hline $\begin{array}{l}\text { Mencionar alguna recomendación que permitiría avanzar en ese proceso tentativo (como, por } \\
\text { ejemplo, formular hipótesis sobre de qué dependerá lo que se busca, pensar y verbalizar lo que se } \\
\text { va a hacer antes de hacerlo, trabajar con símbolos para favorecer la interpretación física, etc.) }\end{array}$ & 0 & 0 & 0 \\
\hline $\begin{array}{l}\text { Hacer alguna referencia a que la forma de introducir los conceptos y principios físicos influye en el } \\
\text { proceso de resolución de problemas }\end{array}$ & 0 & 0 & 0 \\
\hline \multicolumn{4}{|l|}{$\begin{array}{l}\text { Respuestas que expresan recomendaciones probablemente relacionadas con una concepción } \\
\text { de los problemas como ejercicios de aplicación, tales como: }\end{array}$} \\
\hline Realice una lectura comprensiva del enunciado & 19 & 7 & 83,9 \\
\hline Tiene que hacer más problemas & 20 & 7 & 87,1 \\
\hline Estudie bien la teoría antes de hacer problemas & 20 & 5 & 80,6 \\
\hline Tiene que mejorar el manejo del aparato matemático & 20 & 6 & 83,9 \\
\hline
\end{tabular}

Para completar el estudio realizado, en la tabla IV se presenta el análisis de la resolución efectuada por alumnos «con éxito» (los mejor calificados del último curso de secundaria y los que han pasado a la universidad) en el que esperábamos encontrar las mismas pautas con las que han sido enseñados.

Debemos recordar que hemos elegido la opción más desfavorable para nuestra hipótesis: si alguno de los aspectos descritos estaba presente en uno de los dos problemas, se valoraba positivamente. Aun así, la forma de proceder de los alumnos ante los problemas es un claro reflejo (con las carencias más acusadas, lógicamente) de lo encontrado en libros y profesores: la plasmación de los datos, incógnitas y fórmulas desde el primer momento en que comienza la resolución sin realizar siquiera un mínimo planteamiento cualitativo, y la inexistencia de indicadores de un comportamiento tentativo (como la formulación de hipótesis, la expresión previa de una estrategia, analizar los resultados para asegurarse de si son correctos...). Es verdad que un $35 \%$ ha tenido «éxito» ante un enunciado elemental, pero con esa forma de proceder es muy poco probable que lo tuvieran ante un verdadero problema para ellos. De hecho, ¡más de la mitad! han abandonado o realizado una resolución totalmente incorrecta (en ambos problemas). Llama poderosamente la atención que la práctica totalidad de los alumnos no analice los resultados que obtienen. Esto no puede ser porque estén seguros de los mismos (evidentemente no puede ser así, a la vista de los porcentajes de abandono o resoluciones totalmente incorrectas) sino por- que no tienen el hábito de hacerlo: no se les ha enseñado a «enfrentarse» a los problemas, sino que se les han mostrado soluciones explicadas. Desde el punto de vista metodológico y actitudinal, el modelo que han vivido durante su formación supone un obstáculo en vez de una ayuda para enfrentarse a problemas. No debe extrañar, pues, la tendencia a reconocer el problema o abandonar.

\section{CONCLUSIONES Y PERSPECTIVAS}

Comenzamos diciendo que la aspiración de la educación científica actual de «enseñar a los alumnos a enfrentarse a problemas», de desarrollar las destrezas de alto nivel intelectual simultáneamente con el conocimiento de un campo específico se limitaba en realidad a la resolución de problemas de lápiz y papel del final de los temas. Pues bien, tras el análisis realizado, podemos afirmar que $n i$ siquiera en esta parcela se les enseña a enfrentarse a problemas, sino que se les transmite una forma de pensar y actuar sólo posible ante situaciones ya familiares que se pueden resolver sin incertidumbre. No se enseña a resolver problemas sino que se muestran soluciones ya hechas. La ausencia prácticamente total de indicadores de un proceso de resolución de problemas como una tarea abierta, que requiere poner en práctica destrezas características de la forma de producir conocimientos científicos, en textos, supone una evidencia incontestable a favor de nuestra hipótesis crítica. 
Tabla IV

Análisis de la resolución de problemas de física por los alumnos.

\begin{tabular}{|c|c|c|c|}
\hline $\begin{array}{l}\text { Ausencia en los estudiantes de los indicadores de una resolución de problemas de } \\
\text { física coherente con la metodología científica }\end{array}$ & $\begin{array}{l}\mathrm{S}(\mathbf{4 0}) \\
\%\end{array}$ & $\begin{array}{l}\mathrm{U}(\mathbf{8 0}) \\
\%\end{array}$ & $\begin{array}{c}\mathbf{S}+\mathbf{U} \\
\%\end{array}$ \\
\hline \multicolumn{4}{|l|}{ I. Sobre el planteamiento cualitativo } \\
\hline 1. Aparecen datos y fórmulas al inicio de la resolución & 97,5 & 95,0 & 95,8 \\
\hline $\begin{array}{l}\text { 2. No se expresan cualitativamente aspectos de la situación física (una descripción } \\
\text { interpretativa de lo que ocurre) }\end{array}$ & 90,0 & 85,0 & 86,7 \\
\hline $\begin{array}{l}\text { 3. No dejan explícito qué es lo que se busca y en qué condiciones se va a realizar la } \\
\text { resolución }\end{array}$ & 97,5 & 87,5 & 90,8 \\
\hline \multicolumn{4}{|l|}{ II. Sobre la emisión de hipótesis } \\
\hline $\begin{array}{l}\text { 4. No emiten hipótesis sobre los factores de los que puede depender la magnitud bus- } \\
\text { cada y la forma de esta dependencia }\end{array}$ & 100,0 & 100,0 & 100,0 \\
\hline \multicolumn{4}{|l|}{ III. Sobre la elaboración de estrategias } \\
\hline $\begin{array}{l}\text { 5. No expresan en forma explícita una posible estrategia de resolución antes de proce- } \\
\text { der a ésta }\end{array}$ & 100,0 & 100,0 & 100,0 \\
\hline 6. No expresan en forma explícita el carácter tentativo de la estrategia & 100,0 & 100,0 & 100,0 \\
\hline \multicolumn{4}{|l|}{ IV. Sobre la resolución del problema } \\
\hline 7. No hacen la resolución como la puesta en práctica de la estrategia planteada & 100,0 & 100,0 & 100,0 \\
\hline 8. No hacen la resolución fundamentando y verbalizando lo que se va haciendo & 100,0 & 100,0 & 100,0 \\
\hline 9. No hacen una resolución literal antes de introducir valores numéricos & 95,0 & 81,3 & 85,8 \\
\hline \multicolumn{4}{|l|}{ V. Sobre el análisis de los resultados } \\
\hline 10. No interpretan los resultados obtenidos & 100,0 & 96,3 & 97,5 \\
\hline 11. No contrastan (no se duda de) los resultados obtenidos & 100,0 & 100,0 & 100,0 \\
\hline \multicolumn{4}{|l|}{ VI. Sobre la consideración de perspectivas abiertas tras la resolución } \\
\hline $\begin{array}{l}\text { 12. Tras la resolución, no contemplan la posibilidad de abordar nuevas situaciones de } \\
\text { interés }\end{array}$ & 100,0 & 100,0 & 100,0 \\
\hline \multicolumn{4}{|l|}{ VI. Otras situaciones } \\
\hline 13. No es correcto el resultado & 70,0 & 62,5 & 65,0 \\
\hline 14. Abandonan la resolución o es totalmente incorrecta & 55,0 & 51,3 & 52,5 \\
\hline
\end{tabular}

No obstante, no basta con este análisis crítico de la enseñanza habitual. Es necesario mostrar que es posible hacerlo mejor de otra manera, que se puede enseñar a los estudiantes a enfrentarse a problemas (y no a reconocer o abandonar). Como ya hemos señalado, desde principios de los ochenta (Gil y Martínez Torregrosa, 1983) disponemos de un modelo coherente con los indicadores expresados, cuya puesta en práctica en otros países ha producido resultados claramente mejores que la enseñanza habitual en distintas áreas (Gil et al., 1987; Ramírez et al., 1994; Furió y Reyes, 1990) en secundaria.

Por nuestra parte, en los dos últimos años, estamos intentando reproducir en los primeros cursos universitarios los buenos resultados hallados por otros investigadores en física de bachillerato y los resultados de que dispo- nemos son muy positivos. El análisis realizado en este artículo junto con la evidencia probada de cómo el modelo alternativo puede cambiar la situación actual en las aulas de nuestra región abre el camino para la necesaria y urgente formación del profesorado de física, tanto para mejorar el aprendizaje de la propia física como para avanzar en las aspiración de la formación científica que la sociedad demanda.

\section{NOTA}

${ }^{1}$ No hemos incluido, por necesitar un análisis más amplio que el de los problemas de «lápiz y papel», la necesidad de que éstos formen parte de un proceso de resolución de un problema más amplio y relevante, de mayor interés. Los científicos se enfrentan a problemas de interés. 


\section{REFERENCIAS BIBLIOGRÁFICAS}

AAAS (1989). Science for all Americans. A Project 2061 Report on Literacy Goals in Science, Mathematics and Technology. American Association for the Advancement of Science. Disponible también en <http://www.aaas.org/ project $2061 />$.

BLACK, P. (2000). Physics 2000: Physics as it Enters a New Millenium, IUPAP, Paul Black, Gordon Drake and Leonard Jossem (eds.). En línea <http://www.physics.ohio-state.edu/ $\sim$ jossem/IUPAP/P2000.pdf $>$.

BODNER, G. M. y McMILLEN, T. L. (1986). Cognitive restructuring as an early stage in problem solving. Journal of Research in Science Teaching, 23(8), pp. 727-737.

CHALMERS, A. (1984). La ciencia y cómo se elabora. Madrid: Siglo XXI.

FERNÁNDEZ, I., GIL, D., VILCHES, A, VALDÉS, P., CACHAPUZ, A., PRAIA, J. y SALINAS, J. (2002). La superación de las visiones deformadas de la ciencia y la tecnología: un requisito esencial para la renovación de la educación científica. Enseñanza de las Ciencias, 20(3), pp. 477-488. En línea: <http://www.unesco.cl/pagina_ciencia_02/Documentos.htm>.

FURIÓ, C. y REYES, J.V. (1990). O modelo de resoluçao de problemas como investigaçao -sua aplicaçao à Química. Boletim da Sociedade Portuguesa de Química, 41(II), pp. 11-16.

GIL, D., FURIÓ, C., VALDÉS, P., SALINAS, J., MARTÍNEZ-TORREGROSA, J., GUISASOLA, J., GONZÁLEZ, E., DUMAS-CARRÉ, A., GOFFARD, M. y PESSOA DE CARVALHO, A. (1999). ¿Tiene sentido seguir distinguiendo entre aprendizaje de conceptos, resolución de problemas de lápiz y papel y realización de prácticas de laboratorio? Enseñanza de las Ciencias, 17(2), pp. 311-320.

GIL PÉREZ, D. y VILCHES, A. (1999). Problemas de la educación científica en la enseñanza secundaria y la universidad: contra las evidencias. Revista Española de Física, 13(5), pp. 10-15.

GIL, D., DUMAS-CARRÉ, A., CAILLOT, M., MARTÍNEZ TORREGROSA, J. y RAMÍREZ, L. (1988a). La resolución de problemas de lápiz y papel como actividad de investigación. Investigación en la Escuela, 6, pp. 3-20.

GIL, D. y MARTÍNEZ TORREGROSA, J. (1983). A model for problem-solving in accordance with scientific methodology. European Journal of Science Education, 5(4), pp. 447-455.

GIL, D., MARTÍNEZ TORREGROSA, J. y SENENT PERÉZ, F. (1988b). El fracaso en la resolución de problemas de física: una investigación orientada por nuevos supuestos. Enseñanza de las Ciencias, 6(2), pp. 131-146.

GILBERT, G. L. (1980). How do I get the answer? Journal of Chemical Education, 57, pp. 79-81.

HARRÉ, R. (1986). Varieties of realism: A rationale for the natural sciences. Oxford: Brasil Blackwell.

HAYES, J. R. (1981). The complete problem solver. Filadelfia: The Franklin Institute Press.

HUDGINS, B. B. (1966). Cómo enseñar a resolver problemas en el aula. Buenos Aires: Paidós.
JANSWEIJER, W., ELSHOUT, J.J. y WIELINGA, B. (1990), On the multiplicity of learning to solve problems, en Mandl, H., De Corte, E., Bennett, N. y Friedrich, H.F. (eds.). Learning and Instruction: European Research in an International Context, 2(1), pp. 127-145. Oxford: Pergamon Press.

KRULIK, S. y RUDNIK, K. (1980). Problem solving in school mathematics. National council of teachers of mathematics. Virginia: Year Book, Reston.

LEONARD et al. (2002). Resolución de problemas basada en el análisis. Hacer del análisis y del razonamiento el foco de la enseñanza de la física. Enseñanza de las Ciencias, 20(3), pp. 387-400.

LONGBOTTOM, J. E. y BUTLER, P. H. (1999). Why Teach Science? Setting Rational Goals for Science Education. Science Education, pp. 473-492.

MALONEY, D.P. (1994). Research on problem solving: physics, en Gabel, D.L. (ed.). Handbook of research on science teaching and learning, pp. 327-354. Nueva York: Simon \& Schuster.

MARTÍNEZ TORREGROSA, J., DOMĖNECH, J.L. y VERDÚ, R. (1994). Del derribo de ideas al levantamiento de puentes: la epistemología de las ciencias como criterio organizador de la enseñanza de las ciencias física y química. Qurriculum, 6-7 (extra sobre enseñanza de las ciencias), pp. 67-89.

MARTÍNEZ TORREGROSA, J., GIL, D. y MARTÍNEZ, B. (2003). La universidad como nivel privilegiado para un aprendizaje como investigación orientada, en Monereo, C. y Pozo, J. I. (eds.). La universidad ante la nueva cultura educativa, pp. 231-244. Madrid: Síntesis.

NATIONAL SCIENCE EDUCATION STANDARDS (1996). National Committee on Science Education Standards and Assessment. National Research Council.

NATIONAL RESEARCH COUNCIL (2001). Knowing what students know: The science and design of educational assessment, Pellegrino, J., Chudowsky, N. y Glaser, R. (eds). Washington DC: National Academy Press.

OSBORNE, J. F., (1996). Beyond constructivism. Science Education, 80(1), pp. 53-82.

PERALES PALACIOS, F.J. (2000). La resolución de problemas, en Perales Palacios, F. y Cañal de León, P. (eds.). Didáctica de la ciencias experimentales. Teoría y práctica de la enseñanza de las ciencias, pp. 289-306. Alcoy: Marfil.

REIF, F. (1983). Understanding and teaching problem solving in Physics. Recherches en Didactique, pp. 3-53. París: CNRS Editions.

RESNICK, R. y HALLIDAY, D. (1988). Física. Parte I. México: Editorial Continental.

TIBERGHIEN, A., LEONARD JOSSEM, E. y BAROJAS, J. (1998). Connecting research in physics education with teacher education, editado por International Commission on Physics Education (ICPE).

VERDÚ, R., OSUNA, L. y MARTÍNEZ TORREGROSA, J. (2002). Enseñar y aprender en una estructura problematizada. Alambique, 34, pp. 47-55. 


\section{ANEXO I}

Problema resuelto presentado a los profesores para que realicen críticas y objeciones (resolución extraída del libro Física, de Resnick y Halliday)

La rapidez de un automóvil que viaja hacia el este se reduce, en forma uniforme, de $20 \mathrm{~m}$ por segundo a $13 \mathrm{~m}$ por segundo, en una distancia de $80 \mathrm{~m}$.

¿Cuál es la magnitud y la dirección de la aceleración constante?

Escogemos arbitrariamente como $x$ positiva: del oeste hacia el este. Conocemos $x$ y $v_{x}$ y queremos determinar $a_{x}$ sin que intervenga el tiempo. En consecuencia, la ecuación apropiada, según la tabla, es la $v_{x}^{2}=v_{x o}^{2}+2 a_{x}\left(x-x_{o}\right)$. Tenemos que $v_{x}=+13,0 \mathrm{~m} / \mathrm{s}, v_{o x}=+20,0 \mathrm{~m} / \mathrm{s},(x-x)=$ $+80,0 \mathrm{~m}$. De la ecuación, obtenemos:

$$
\begin{aligned}
& a_{x}=\left(v_{x}^{2}-v_{x o}^{2}\right) / 2\left(x-x_{o}\right) \\
& a_{x}=\left((13,0 \mathrm{~m} / \mathrm{s})^{2}-(20,0 \mathrm{~m} / \mathrm{s})^{2}\right) / 2(80,0 \mathrm{~m}) \\
& a_{x}=-1,44 \mathrm{~m} / \mathrm{s}^{2}
\end{aligned}
$$

La dirección de la aceleración es hacia el oeste. El automóvil está frenado y se mueve hacia el este. Cuando la rapidez está disminuyendo, con frecuencia se dice que está desacelerando.

¿Cuánto tiempo ha transcurrido durante esta desaceleración?

Si sólo se usan los datos originales, se puede ver en la tabla que la ecuación $x=x_{o}+1 / 2\left(v_{x o}+v_{x}\right) t$ es la apropiada.

$$
\begin{aligned}
& t=2\left(x-x_{o}\right) /\left(v_{x o}+v_{x}\right) \\
& t=2(80,0 \mathrm{~m}) /(20,0+13,0) \mathrm{m} / \mathrm{s} \\
& t=4,85 \mathrm{~s}
\end{aligned}
$$

\section{ANEXO II}

Problema propuesto a profesores de física de secundaria y universitarios chilenos para su «resolución didáctica ideal»

Una camioneta transporta un cajón con manzanas. El cajón va sobre la plataforma sin ningún tipo de amarre. El coeficiente de rozamiento

cinético y estático entre la plataforma y el cajón es 0,50 y 0,70 , respectivamente.

¿Cuál es la máxima aceleración con que puede arrancar la camioneta en un semáforo sobre una calle horizontal de forma que el cajón no se deslice sobre la plataforma? 